\title{
There Is No Global Running Pattern More Economic Than Another at Endurance Running Speeds
}

\author{
Aurélien Patoz, Thibault Lussiana, Bastiaan Breine, Cyrille Gindre, and Kim Hébert-Losier
}

\begin{abstract}
Purpose: The subjective Volodalen® score (V®score) and the objective duty factor metric can both assess global running patterns. The authors aimed to investigate the relation between running economy (RE) at endurance running speeds and the global running pattern quantified using both subjective and objective measures. Methods: RE and 3-dimensional whole-body kinematics were acquired by indirect calorimetry and an optoelectronic system, respectively, for 52 trained runners during treadmill runs at 10, 12, and $14 \mathrm{~km} / \mathrm{h}$. Results: Correlations between RE and V®score and RE and duty factor were negligible and nonsignificant across speeds tested $(P \geq .20)$, except for a low and significant correlation between $\mathrm{RE}$ and $\mathrm{V} \circledast \mathrm{score}$ at $10 \mathrm{~km} / \mathrm{h}$. Conclusions: These findings suggest there is no global running pattern more economic than another at endurance running speeds. Therefore, there is no advantage of choosing, favoring, or prescribing one specific global running pattern along a continuum based on $\mathrm{V} ®$ score or duty factor metrics, and coaches should not try to modify the spontaneous running pattern of runners at endurance running speed to improve RE.
\end{abstract}

Keywords: gait analysis, motion analysis, biomechanics, duty factor, Volodalen® score

As early as 1985 , the running pattern was suggested to be multifactorial, and foot placement, arm swing, body angle, rear leg lift, and stride length should be considered together. ${ }^{1}$ Following this concept, a subjective method allowing global definition of selfselected running patterns was created. ${ }^{2}$ This method considers runners as global systems in which the change or alteration of one variable is likely to affect another. Runners are classified based on visual observations of 5 key elements where each element is scored on a 1 to 5 scale. The 5 individual scores are summed to provide a global score, the Volodalen ${ }^{\circledR}$ score (V®score), ranging from 5 to $25 .^{2}$ The resulting subjectively based score is used to classify runners in 2 groups: aerial (V®score $>15$ ) and terrestrial $(\mathrm{V} \circledast$ score $\leq 15) .^{2}$ The authors observed that runners in each group present similar running economy (RE) characteristics but employ different strategies to lower their energetic cost, with terrestrial runners relying more on forward propulsion strategies and aerial runners more on rebound strategies. ${ }^{2}$

Runners have also been categorized in 2 groups using the duty factor (DF), ${ }^{3,4}$ that is, an objective variable representing the ratio of ground contact time $\left(t_{c}\right)$ to stride time $\left[t_{c}+\right.$ swing time $\left.\left(t_{s}\right)\right]$, with a higher DF reflecting a greater relative contribution of $t_{c}$ to the running stride. ${ }^{5} \mathrm{DF}$ was thought to be representative of a global biomechanical behavior, considering the duration of force

Patoz is with the Inst of Sport Sciences, University of Lausanne, Lausanne, Switzerland. Patoz, Lussiana, Breine, and Gindre are with the Research and Development Dept, Volodalen Swiss Sport Lab, Aigle, Switzerland. Lussiana and Gindre are with the Research and Development Dept, Volodalen, Chavéria, France. Lussiana is also with the Research Unit EA3920 Prognostic Markers and Regulatory Factors of Cardiovascular Diseases and Exercise Performance, Health, Innovation Platform, University of Bourgogne Franche-Comté, Besançon, France. Breine is also with the Dept of Movement and Sports Sciences, Ghent University, Ghent, Belgium. Hébert-Losier is with the Div of Health, Engineering, Computing and Science, Te Huataki Waiora School of Health, University of Waikato, Adams Centre for High Performance, Tauranga, New Zealand, and the Dept of Sports Science, National Sports Inst of Malaysia, Kuala Lumpur, Malaysia. Patoz (aurelien@volodalen.com) is corresponding author. production $\left(t_{c}\right)$ in relation to stride duration. ${ }^{3,4}$ Runners with a low DF exhibited a more symmetrical running step, anterior (midfoot and forefoot) strike pattern, and extended lower limb during $t_{c}$ than runners with a high $\mathrm{DF}$, whereas the latter runners exhibited greater lower limb flexion during $t_{c}$, more rearfoot strike pattern, and less work against gravity to generate forward propulsion. ${ }^{3,4}$ Therefore, runners with high and low DF values used different running strategies. Despite these kinematic differences, the 2 groups demonstrated similar RE values, indicating the 2 strategies are energetically equivalent at endurance running speeds. ${ }^{3}$

Given that the V®score and DF are continuous variables, the global running pattern of individuals could be defined along a continuum rather than categorized into 2 or more groups. However, the relation between $\mathrm{RE}$ and global running patterns along a continuum has, to the best of our knowledge, not been investigated so far. Hence, we aimed to investigate the relation between RE and global running pattern, assessed using either the subjective V®score or the objective DF metric. We hypothesized that RE would not be related to either $\mathrm{V} \circledast$ score or DF.

\section{Methods}

\section{Subjects}

A total of 52 trained runners, 31 males (mean [SD]; age $=31$ [8] y, height $=174[7] \mathrm{cm}$, body mass $=66[10] \mathrm{kg}, 21.1 \mathrm{~km}$ recent running performance $=91$ [9] $\min$, running experience $=8[6] \mathrm{y}$, weekly running distance $=55[19] \mathrm{km}$ ) and 21 females (age $=32$ [9] y, height $=162[4] \mathrm{cm}$, body mass $=52[5] \mathrm{kg}, 21.1 \mathrm{~km}$ recent running performance $=102$ [9] min, running experience $=7$ [4] y, weekly running distance $=50[21] \mathrm{km}$ ) participated in this study. Participants were required to be in good self-reported general health, with no current or recent $(<3$ mo) musculoskeletal injuries, and to have competed in a road race in the last year with finishing times of $10 \mathrm{~km} \leq 50 \mathrm{~min}, 21.1 \mathrm{~km} \leq 110 \mathrm{~min}$, or $42.2 \mathrm{~km} \leq 230 \mathrm{~min}$. The ethical committee of the National Sports Institute of Malaysia 
approved the study protocol (ISNRP: 26/2015), which adhered to the latest Declaration of Helsinki.

\section{Design}

Each participant completed one experimental laboratory session. After providing written informed consent, participants ran 5 minutes at $9 \mathrm{~km} / \mathrm{h}$ on a treadmill $\left(\mathrm{h} / \mathrm{p} / \operatorname{cosmos}\right.$ mercury ${ }^{\circledR} ; \mathrm{h} / \mathrm{p} /$ cosmos sports \& medical gmbh, Nussdorf-Traunstein, Germany) as warm-up. Participants then completed three 4-minute runs at 10,12 , and $14 \mathrm{~km} / \mathrm{h}$ (with 2-min recovery between runs) on the treadmill, during which time RE was assessed (Appendix A). RE was expressed as the oxygen cost per mass to the power of 0.75 per kilometer (in milliliter per kilometer per kilogram ${ }^{0.75}$ ) to minimize the influence of body mass per se on oxygen consumption during running. ${ }^{6} \mathrm{~A}$ higher RE value indicates a less economical runner.

Retroreflective markers were subsequently positioned on individuals to assess running kinematics (Appendix B). For each participant, a 1-second static calibration trial was recorded, which was followed by three 30 -second runs at 10,12 , and $14 \mathrm{~km} /$ $\mathrm{h}$ (with 1-min recovery between runs) to collect 3-dimensional (3D) kinematic data in the last 10 seconds segment of these runs. $\mathrm{RE}$ and biomechanics were assessed separately given laboratory constraints and interference with data quality (eg, presence of testing equipment that occluded markers). All participants were familiar with running on a treadmill as part of their usual training programs and wore their habitual running shoes during testing (shoe mass $=223[36] \mathrm{g}$, shoe stack height $=25[3] \mathrm{mm}$, and shoe heel-to-toe drop $=8[3] \mathrm{mm}$ ).

\section{Methodology}

The 5 elements of the $\mathrm{V} \circledast$ score were objectively retrieved from 3D kinematic data (Appendix C). Each element was converted to a score from 1 to 5 using the 20th, 40th, 60th, 80th, and 100th percentiles. The $\mathrm{V} \circledast$ score was subsequently obtained by summing these 5 objectively measured elements.

$t_{s}$ and $t_{c}$ were defined as the time from toe-off to foot strike and from foot strike to toe-off of the same foot, respectively, to calculate DF as:

$$
\mathrm{DF}=\frac{t_{c}}{t_{c}+t_{s}} .
$$

The biomechanical variables extracted from the 10-second data collection for each participant were averaged for subsequent statistical analyses.

\section{Statistical Analysis}

Descriptive statistics are presented using mean (SD). As all data were normality distributed based on Kolmogorov-Smirnov tests $(P \geq .08)$, we extracted Pearson correlation coefficients $(r)$ with 95\% confidence intervals and corresponding $P$ values to explore the relationship between RE and V®score and RE and DF at the 3 running speeds separately. Correlations were considered very high, high, moderate, low, and negligible when absolute $r$ values were between .90 and $1.00, .70$ and $.89, .50$ and $.69, .30$ and .49 , and .00 and .29 , respectively. ${ }^{9}$ Statistical analyses were performed using Jamovi (version 1.6.23; https://www.jamovi.org) with a level of significance set at $P \leq .05$.

\section{Results}

Correlations between RE and V®score and between RE and DF were negligible and nonsignificant at all tested speeds $(|r| \leq .18$; $P \geq .20$ ), except for a low and significant correlation between RE and $\mathrm{V} \circledast$ score at $10 \mathrm{~km} / \mathrm{h}(r=.30 ; P=.03$; Figure 1$)$.

\section{Discussion}

In line with our hypothesis, RE was not related to either V®score or DF, except at $10 \mathrm{~km} / \mathrm{h}$ where a low correlation was observed between RE and $\mathrm{V} \circledast$ score. This single low correlation suggests little clinical relevance and a potential spurious finding (Figure 1A). Similarly, no significant relation between RE and DF was detected (Figure 1B). This suggests that runners with a global running pattern at either ends of the subjective spectrum (most terrestrial, $\mathrm{V} \bowtie s c o r e=5$; vs most aerial, $\mathrm{V} ® s c o r e=25$ ), or objective DF spectrum (highest vs lowest DF) exhibit similar RE at endurance running speeds. Therefore, there is no advantage of choosing, favoring, or prescribing one specific global running pattern along a continuum based on V®score or DF metrics. These results confirm previous findings of similar RE between runners categorized as terrestrial and aerial, ${ }^{2}$ or as high and low DF runners. $^{3}$

The subconscious fine-tuning of running biomechanics, known as self-optimization, was first introduced by Williams and Cavanagh ${ }^{10}$ and extended upon by Moore. ${ }^{11}$ The concept of self-optimization might explain the null relationship found between $\mathrm{RE}$ and global running pattern. Indeed, RE was proposed to result from a weighted influence of several variables. ${ }^{10}$ Moreover, runners appear to naturally adopt a running biomechanics (eg, stride length, stride frequency, contact time, leg stiffness) that is energetically optimal, or at least near optimal. ${ }^{11,12}$ This altogether suggests that the relative importance of these underlying variables might differ along the spectrum of global running patterns as defined using the V®score or DF metric but that all these different global running patterns lead to a similar RE. Determining the global running pattern of a runner might nonetheless inform which variables are contributing the most to RE and not yet optimized for a given individual. Hence, slight alterations in suboptimal and interconnected variables might confer RE advantages at an individual level, notwithstanding that most biomechanical fine-tuning to improve RE may have already occurred in trained and experienced runners. ${ }^{11}$

\section{Practical Applications}

There is no advantage of choosing, favoring, or prescribing one specific global running pattern along a continuum based on V®score or DF metrics. Therefore, coaches should not try to modify the spontaneous running pattern of runners at endurance running speed to improve RE.

\section{Conclusions}

The current study findings suggest there is no significant or meaningful relation between RE and global running pattern, assessed using either the V®score or the DF metric, meaning that any spontaneous self-selected running style might be energetically optimal. In other words, there is no global running pattern more economic than another at endurance running speeds. 

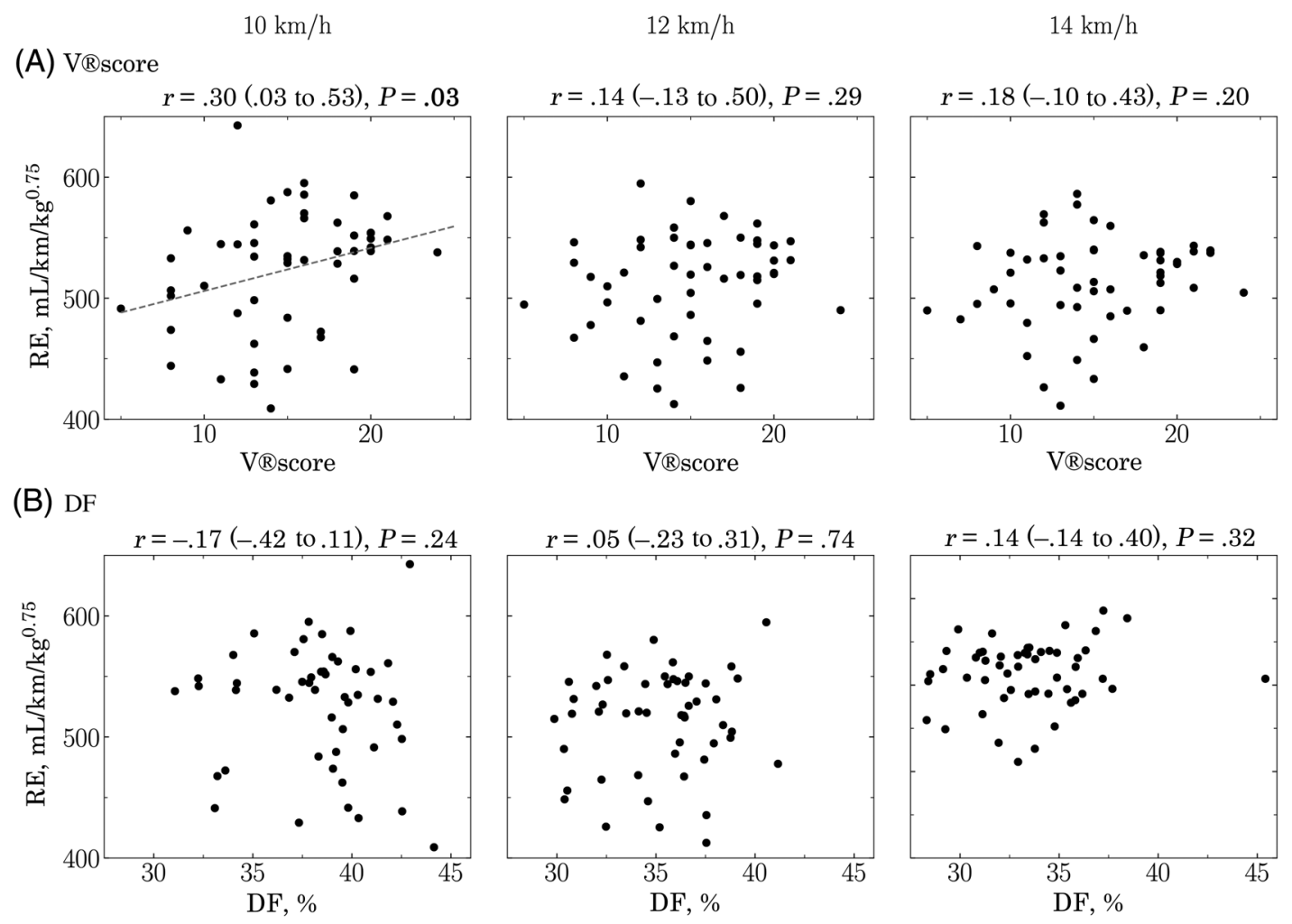

Figure $1-\mathrm{RE}$ as function of (A) V®score and (B) DF at 3 different running speeds for 52 participants. Statistical significance $(P \leq .05)$ is indicated in bold. The gray dashed line between RE and V®score at $10 \mathrm{~km} / \mathrm{h}$ represents a significant but low Pearson correlation. DF indicates duty factor; $r$, Pearson correlation coefficient together with its $95 \%$ CIs (lower and upper); RE, running economy; V®score, Volodalen ${ }^{\circledR}$ score.

\section{Acknowledgments}

The authors thank Mr. Chris Tee Chow Li for assistance during the data collection process. The authors also thank the participants for their time and participation. This study was financially supported by the University of Bourgogne Franche-Comté (France) and the National Sports Institute of Malaysia. C.G. is the originator of the Volodalen ${ }^{\circledR}$ scale. However, this paper does not constitute endorsement of the scale by the other authors and stems completely from a $\mathrm{PhD}$ research project undertaken at the FrancheComté University by T.L. The data set supporting this article is available on request to the corresponding author. Conceptualization was done by T.L., C.G., and K.H.-L.; methodology by T.L., C.G., and K.H.-L.; investigation was by T.L. and K.H.-L.; formal analysis by T.L., A.P., B.B., and K.H.-L.; writing—original draft preparation—by A.P. and B.B.; writing—review and editing-by A.P., T.L., B.B., C.G., and K.H.-L.; supervision was by K.H.-L; and funding acquisition was done by T.L. and K.H.-L.

\section{References}

1. Subotnick SI. The biomechanics of running. Implications for the prevention of foot injuries. Sports Med. 1985;2(2):144-153. PubMed ID: 2860714 doi:10.2165/00007256-198502020-00006

2. Lussiana T, Gindre C, Hébert-Losier K, Sagawa Y, Gimenez P, Mourot L. Similar Running economy with different running patterns along the aerial-terrestrial continuum. Int J Sports Physiol Perform. 2017;12(4):481. PubMed ID: 27617625 doi:10.1123/ijspp.2016-0107

3. Lussiana T, Patoz A, Gindre C, Mourot L, Hébert-Losier K. The implications of time on the ground on running economy: less is not always better. J Exp Biol. 2019;222(6):jeb192047. doi:10.1242/jeb. 192047

4. Patoz A, Lussiana T, Thouvenot A, Mourot L, Gindre C. Duty factor reflects lower limb kinematics of running. Appl Sci. 2020;10(24): 8818.

5. Minetti AE. A model equation for the prediction of mechanical internal work of terrestrial locomotion. J Biomech. 1998;31(5):463-468. PubMed ID: 9727344 doi:10.1016/S0021-9290(98)00038-4

6. Svedenhag J, Sjödin B. Body-mass-modified running economy and step length in elite male middle- and long-distance runners. Int $J$ Sports Med. 1994;15(6):305-310. PubMed ID: 7822068 doi:10. 1055/s-2007-1021065

7. Altman AR, Davis IS. A kinematic method for footstrike pattern detection in barefoot and shod runners. Gait Posture. 2012;35(2): 298-300. PubMed ID: 22075193 doi:10.1016/j.gaitpost.2011.09.104

8. Lussiana T, Gindre C, Mourot L, Hébert-Losier K. Do subjective assessments of running patterns reflect objective parameters? Eur J Sport Sci. 2017;17(7):847-857. PubMed ID: 28488928 doi:10.1080/ 17461391.2017.1325072

9. Hinkle DE, Wiersma W, Jurs SG. Applied Statistics for the Behavioral Sciences. 5th ed. Boston, MA: Houghton Mifflin; 2002.

10. Williams KR, Cavanagh PR. Relationship between distance running mechanics, running economy, and performance. J Appl Physiol. 1987;63(3):1236-1245. PubMed ID: 3654469 doi:10.1152/jappl. 1987.63.3.1236

11. Moore IS. Is there an economical running technique? A review of modifiable biomechanical factors affecting running economy. Sports Med. 2016;46(6):793-807. PubMed ID: 26816209 doi:10.1007/ s40279-016-0474-4 
12. Moore IS, Ashford KJ, Cross C, Hope J, Jones HSR, McCarthyRyan M. Humans optimize ground contact time and leg stiffness to minimize the metabolic cost of running. Front Sports Act Living. 2019;1:53. PubMed ID: 33344976 doi:10.3389/fspor.2019.00053

\section{Appendix A: Gas Exchange Analysis}

Gas exchange was measured using TrueOne 2400 (ParvoMedics, Inc, Sandy, UT) during the three 4-minute runs. Prior to the experiment, the gas analyzer was calibrated using ambient air $\left(\mathrm{O}_{2}: 20.93 \%\right.$ and $\left.\mathrm{CO}_{2}: 0.03 \%\right)$ and a gas mixture of known concentration $\left(\mathrm{O}_{2}: 16.00 \%\right.$ and $\left.\mathrm{CO}_{2}: 4.001 \%\right)$. Volume calibration was performed at different flow rates with a 3-L calibration syringe (5530 series; Hans Rudolph, Shawnee, KS). Oxygen consumption, carbon dioxide production, and respiratory exchange ratio values were averaged over the last minute of each 4-minute run. Steady state was confirmed through visual inspection of the oxygen consumption and carbon dioxide production curves. Respiratory exchange ratio had to remain below unity during the trials for data to be included in the analysis; otherwise, the corresponding data were excluded as deemed to not represent a submaximal effort. No trial was excluded on this basis.

\section{Appendix B: Kinematic Analysis}

The 3D kinematic data were collected at $200 \mathrm{~Hz}$ using 7 infrared Oqus cameras (5 Oqus 300+, one Oqus 310+, and one Oqus 311+) and Qualisys Track Manager software (version 2.1.1) build 2902 together with the Project Automation Framework Running package (version 4.4; Qualisys AB, Göteborg, Sweden). A virtual laboratory coordinate system was generated such that the $x-y-z$ axes denoted the mediolateral (pointing toward the right side of the body), posterior-anterior, and inferior-superior directions, respectively. A total of 35 retroreflective markers of $12 \mathrm{~mm}$ in diameter were used for static calibration and dynamic running trials and were affixed to the skin and shoes of individuals over anatomical landmarks using double-sided tape following standard guidelines from the Project Automation Framework Running package, as already reported elsewhere. ${ }^{3}$ The $3 \mathrm{D}$ marker data were exported in .c3d format and processed in Visual3D Professional software (version 5.02.25; C-Motion Inc, Germantown, MD). More explicitly, the 3D marker data were interpolated using a third-order polynomial least-square fit algorithm, allowing a maximum of 20 frames for gap filling, and subsequently low-pass filtered at $20 \mathrm{~Hz}$ using a fourth-order Butterworth filter.
From the marker set, a full-body biomechanical model with 6 degrees of freedom at each joint and 15 rigid segments was constructed. The model included the head, upper arms, lower arms, hands, thorax, pelvis, thighs, shanks, and feet. Segments were assigned inertial properties and center of mass (COM) locations based on their shape and relative mass. Kinematic parameters were calculated using rigid-body analysis and wholebody COM location was calculated from the parameters of all 15 segments.

Running events were derived from the trajectories of the 3D marker data using similar procedures to those previously reported elsewhere. ${ }^{3}$ More explicitly, a midfoot landmark was generated midway between the heel and toe markers. Foot-strike was defined as the instance when the midfoot landmark reached a local minimal vertical velocity prior to it reaching a peak vertical velocity reflecting the start of swing. Toe-off was defined as the instance when the toe marker attained a peak vertical acceleration before reaching a $7-\mathrm{cm}$ vertical position. All events were verified to ensure correct identification and were manually adjusted when required.

\section{Appendix C: Objective Measure of the 5 Elements of the V®score}

The range of vertical displacement of the head COM, range of horizontal displacement of the elbow joint centers (ie, minimum to maximum horizontal position during each cycle), vertical position of the pelvis COM at foot strike, horizontal distance between the heel marker and pelvis COM at foot strike, and foot strike angle at ground contact were extracted. The first 4 elements were normalized to participants' height. The foot strike angle was normalized to the foot-ground angle recording during the static trial following procedures described in Altman and Davis. ${ }^{7}$ These objective measures extracted from 3D data largely relate to their subjective Volodalen ${ }^{\circledR}$ counterparts ${ }^{8}$ and were herein used to calculate the $\mathrm{V} \circledast$ score. 